\title{
Towards a Fuzzy Logic Model for Chemical Risk Assessment by Inhalation in Laboratories
}

\author{
Oscar M. Obando R. and Cesar Garita, Costa Rica Institute of Technology, Costa Rica
}

\begin{abstract}
This paper presents a computational Fuzzy Logic Model for chemical hazards risk assessment by inhalation in laboratories. Some influence factors on chemical risk are deterministic but not completely measurable. As a result, this research focusses on Fuzzy Logic, instead of a probabilistic approach. Data for simulation is based on a hypothetical but completely realistic laboratory. Dependency on time has been identified as an important factor, describing events on the laboratory installation. Exposure levels for individual factors, labor hours and general health overcome current known strategies used on risk assessment. These conditions are included in the proposed model.
\end{abstract}

Index Terms-Chemical Risk, Computational Modeling, Fuzzy Logic, Human Factors, Inhalation Risk, Risk analysis, Risk Assessment, Time Dependency.

\section{INTRODUCCIÓN}

$\mathrm{E}^{\mathrm{se}}$ ste artículo propone la utilización de un enfoque computacional basado en lógica difusa para el cálculo y modelado del riesgo químico por inhalación (RQI) en un ambiente de laboratorio en el que existe manipulación de sustancias. Varias características del problema hacen de la lógica difusa un enfoque más apropiado para la valoración del RQI en contraste con los métodos clásicos de cálculo determinístico [1]. El presente estudio es realizado como parte de la tesis para optar por el grado de Maestría en Ciencias de la Computación en el Instituto Tecnológico de Costa Rica.

La valoración del RQI es la identificación y cuantificación del riesgo resultante de la exposición de determinado agente(s) químico con cierto grado de peligrosidad a uno o varios individuos. La peligrosidad es una característica propia de las propiedades físico-químicas de la sustancia y la exposición es una cuantificación de la cantidad, tiempo y concentración que tiene una sustancia en condiciones cercanas a un individuo [2]. El método de valoración cualitativo está basado únicamente en la conformidad de reglas de seguridad preestablecidas. La técnica semicuantitativa persigue caracterizar la ocurrencia de consecuencias indeseables (accidentes o sobreexposiciones) y la probabilidad de éstas para obtener un índice numérico que indique el grado de riesgo existente. Finalmente, el método cuantitativo se refiere a una medición directa en el sitio bajo análisis [3]. La técnica cuantitativa implica la utilización de equipos y reactivos a un costo elevado, mientras que la técnica

Óscar M. Obando R., Maestría en Computación, Instituto Tecnológico de Costa Rica, Apdo. 159-7050, Cartago, Costa Rica (e-mail: ing.oscar.obando@gmail.com).

Cesar Garita, Maestría en Computación, Instituto Tecnológico de Costa Rica, Apdo. 159-7050, Cartago, Costa Rica (e-mail: cesar@itcr.ac.cr). cualitativa no representa de manera fidedigna muchos de los factores incidentes en el riesgo bajo estudio. De esta manera, el enfoque semi-cuantitativo es el método con mayor posibilidad de impacto en los enfoques de gestión del RQI [4].

En este trabajo se propone la aplicación del modelo para tomar en cuenta factores de naturaleza distinta, desde la concentración estimada de partículas suspendidas en la atmósfera del laboratorio, hasta la condición de salud de la personas que son expuestas. El impacto de la metodología propuesta busca sistematizar la estimación de RQI permanente en la instalación, identificar de manera preventiva los factores de mayor peso y permitir gestionar los recursos de medidas de seguridad en los puntos de mayor prioridad.

En la sección de trabajos relacionados se hace una breve revisión del estado actual del tema. En la sección de conceptos, se definen los umbrales límite de exposición y la lógica difusa. En la descripción general del enfoque, se explica cuál fue el procedimiento seguido. En la sección de metodología de desarrollo se expone un ejemplo de uso y el detalle matemático del modelado utilizado. En la sección de resultados se resumen los principales hallazgos del estudio. Finalmente, se presentan los lineamientos para la continuación del tema, en la sección de trabajo futuro y las conclusiones de la investigación.

\section{TRABAJOS RELACIONADOS}

Algunos países como España, tienen un conjunto de normas basadas en estudios científicos para la atención del RQI. El Instituto Nacional de Seguridad e Higiene en el Trabajo del Ministerio de Empleo (INSHT) tiene una colección abundante de normas técnicas de protección [5], [6].

Los modelos modernos de evaluación de riesgo surgieron en 1999 [7]. El referente es el emitido por el "Health and Safety Executive" (HSE) del Reino Unido. El Departamento de salud y Seguridad del Ministerio de Trabajo, Asuntos Sociales y Empleo de Holanda desarrolló una herramienta computacional para el análisis de riesgo de productos químicos por inhalación [8].

En [3] se propone el uso de una matriz de riesgo difuso para caracterizar un proceso que ha sido identificado en varias dimensiones físicas. Un enfoque similar es propuesto en [9]. Varias características hacen de la lógica difusa un enfoque recomendable para la valoración de riesgo en contraste con los métodos clásicos de cálculo determinístico [1]. Este marco puede ser aplicado en muchas áreas como en clasificación de patrones [10]. La lógica difusa corresponde a una forma de lidiar con problemas en los que la imprecisión es la ausencia 
de un criterio de frontera definido de pertenencia a un conjunto [11]. La lógica difusa debe ser candidata para ser utilizada en el análisis de este tipo de fenómenos [12]. En otras aplicaciones destacan la evaluación de las incertidumbres de varios indicadores de riesgo en una aplicación de suelos restaurados [13] y el transporte de materiales peligrosos [14].

El enfoque propuesto corresponde a una lógica difusa en la que los valores límite de exposición, los índices de peligrosidad de las sustancias, los factores de estado de las medidas de seguridad en las instalaciones, las características de las personas y el carácter dinámico de las condiciones de un laboratorio químico, serán tomados en cuenta para determinar el RQI individual y global de manera semi-cuantitativa.

\section{CONCEPTOS}

Esta sección define de manera muy breve dos elementos básicos en la investigación: los valores límite de exposición y la lógica difusa.

\section{A. Valores de Umbral Límite}

Los Valores de Umbral Límite (TLV, por sus siglas en inglés) se refieren a las concentraciones de sustancias químicas y representan condiciones bajo las cuales se cree que las personas pueden exponerse repetidamente, día tras día, durante su vida laboral sin sufrir efectos adversos a su salud [15]. Los TLV se relacionan únicamente con riesgo por inhalación.

Los TLV-TWA corresponden a la concentración permitida promedio ponderada en el tiempo de una sustancia, para una jornada laboral de 8 horas y una semana laboral de 40 horas. Los TLV-STEL corresponden a una exposición máxima promedio ponderada en el tiempo de 15 minutos del TWA que no debe ser excedida en ningún momento [15].

\section{B. Lógica Difusa}

La lógica difusa es un conjunto de principios matemáticos, basados en grados de pertenencia. Este enfoque refleja la semántica clasificadora del pensamiento humano e intenta modelar el significado de las palabras utilizadas para ello, así como el proceso de toma de decisiones y sentido común [16].

La estructura básica de un sistema de inferencia difusa puede ser observada en la Fig. 1. Consiste de tres componentes principales: una base de reglas, un conjunto de funciones de pertenencia en el fuzificador y un mecanismo de razonamiento que se aplica para derivar una salida razonable.

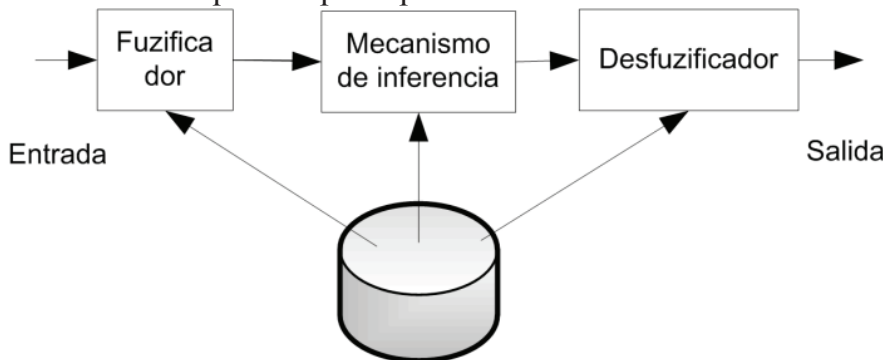

Funciones de pertenencia y reglas

Fig. 1. Estructura base de un sistema difuso.

\section{DESCRIPCIÓN GENERAL DEL ENFOQUE}

En el presente estudio se procedió a determinar los factores principales que se toman en cuenta en la valoración de RQI. Se ha identificado a las sustancias químicas, las cantidades en la atmósfera de los laboratorios provocadas por los eventos que generan emanación de los agentes, las condiciones de salud, sensibilidad química, edad, y tiempo de exposición de las personas.

Cada factor es especificado de manera matemática, para introducirlo en un modelo de lógica difusa. Este tiene como objetivo determinar de manera cuantificable el RQI tanto para las personas de manera individual como para la instalación. El RQI es expresado como función del tiempo, dependiendo de la realización de los procedimientos de laboratorio.

Se utiliza un conjunto de pruebas simplificado, que propone el análisis de cinco procedimientos típicos de un laboratorio de análisis químico.

El modelo es sometido a un conjunto de pruebas generadas de manera aleatoria, respetando la frecuencia en la que se espera la realización de los eventos respectivos. Asimismo, se toma en cuenta la hipotética participación de seis personas que laboran en distintas jornadas en la instalación. Cada persona posee características distintas. Los resultados son obtenidos con una calendarización correspondiente a 15 semanas de trabajo en el laboratorio. Tanto las pruebas como el modelo fueron implementados en el ambiente computacional $\mathrm{R}$.

\section{Metodología de Desarrollo}

En esta sección se muestra un conjunto de datos de prueba basado en un caso real de aplicación y el modelo de cálculo utilizado en esta investigación.

\section{A. Conjunto de Datos de Ejemplo}

El conjunto de datos de prueba presenta una condición hipotética, pero completamente factible de una situación real. Un laboratorio químico es una entidad en la que existe un conjunto de sustancias químicas, que es alterado por una serie de eventos (procedimientos de laboratorio) con una duración y frecuencia dadas. Existe, la liberación de cierta cantidad de moléculas suspendidas en el aire expresadas en partes por millón (PPM). A su vez, un conjunto de personas con diversas condiciones, experimentan una exposición diaria determinada.

En la Tabla I se detallan cinco eventos típicos que se efectúan en un laboratorio de análisis de gases. Se muestran los agentes liberados, la cantidad de partículas por millón, la duración y la frecuencia semanal o mensual del mismo. En la Tabla II se muestran los atributos de seis personas que frecuentan el sitio del laboratorio de interés. En ésta última tabla, la condición general de salud está indicada en una escala de 1 (muy buena) a 10 (muy mala). Otro de los elementos de importancia es la hipersensibilidad que han mostrado estos individuos a las sustancias respectivas. 
TABLA I

Detalle de eventos.PPM: partes por millón, Frec.:frecuencia

\begin{tabular}{|l|l|l|l|}
\hline Evento & Agente & PPM & Frec. \\
\hline \hline \multirow{2}{*}{$\begin{array}{l}\text { Disol. Fenol en etanol al 10\% } \\
\text { (20 minutos) }\end{array}$} & Fenol & 7.65 & \multirow{2}{*}{$3 / \mathrm{mes}$} \\
\cline { 2 - 3 } & Etanol & 39.07 & \\
\hline \multirow{2}{*}{$\begin{array}{l}\text { Análisis Amoniáco } \\
\text { (3 horas) }\end{array}$} & Fenol & 153.01 & \multirow{2}{*}{$1 / \mathrm{sem}$} \\
\cline { 2 - 3 } & Etanol & 468.85 & \\
\cline { 2 - 3 } & Acetona & 31.03 & \\
\hline \multirow{3}{*}{$\begin{array}{l}\text { Análisis HPLC } \\
\text { (5 horas) }\end{array}$} & Metanol & 374.53 & \multirow{2}{*}{$2 / \mathrm{sem}$} \\
\cline { 2 - 3 } & ACN & 263.09 & \\
\cline { 2 - 3 } & Hexano & 278.49 & \\
\cline { 2 - 3 } & $\begin{array}{l}\text { Ácido } \\
\text { tri cloro } \\
\text { acético }\end{array}$ & 73.44 & \\
\hline \multirow{2}{*}{$\begin{array}{l}\text { Análisis Mercurio Búnker } \\
\text { (3 horas) }\end{array}$} & Mercurio & 0.36 & \\
\cline { 2 - 3 } & $\begin{array}{l}\text { Ácido } \\
\text { per } \\
\text { clórico }\end{array}$ & 37.89 & \\
\hline $\begin{array}{l}\text { Disol. Ácido Clorhídrico (90 } \\
\text { minutos) }\end{array}$ & $\begin{array}{l}\text { Ácido } \\
\text { clor } \\
\text { hídrico }\end{array}$ & 394.95 & \multirow{2}{*}{$2 / \mathrm{mes}$} \\
\hline \hline
\end{tabular}

TABLA II

Detalle de personas.G: género, E: edad, L: años de laborar en la actividad química, $\mathrm{X}$ : permanencia diaria en el laboratorio (horas), S: estado de salud

\begin{tabular}{|l|l|l|l|l|l|}
\hline Persona & G & E & L & X & S \\
\hline \hline Persona 1 & M & 35 & 8 & 2 & 1 \\
\hline Persona 2 & M & 37 & 6 & 3 & 6 \\
\hline Persona 3 & F & 23 & 1 & 8 & 2 \\
\hline Persona 4 & M & 22 & 1 & 3 & 2 \\
\hline Persona 5 & F & 27 & 3 & 6 & 5 \\
\hline Persona 6 & F & 55 & 10 & 2 & 6 \\
\hline \hline
\end{tabular}

La sensibilidad se manifiesta con cambios en la reacción ante la exposición del agente como por ejemplo irritación en la piel, mucosa nasal, ojos y vías respiratorias superiores [17]. En la Tabla III se muestra la hipersensibilidad del personal expuesto a las sustancias de interés. La hipersensibilidad en este caso, se cuantifica en una escala de 1 a 5 , donde 1 corresponde a una reacción nula y un 5 a una reacción que requiere atención médica inmediata.

1) Detalles adicionales de los datos de prueba:

- Cada uno de los agentes generados por los eventos de la Tabla I existe en la atmósfera de la instalación debido a dos fuentes: estacionaria y transitoria. La fuente estacionaria corresponde a la concentración de moléculas en el laboratorio debido al inventario existente; se asume constante para efectos de este ejemplo. La fuente transitoria es provocada por la ocurrencia de los eventos indicados y describe los cambios de concentración de los químicos en el sitio.

- Cada uno de los eventos mostrados en la Tabla I es calendarizado para su simulación en determinados instantes, a lo largo de un periodo de 15 semanas.

- El estado de las medidas de seguridad no es tomado en cuenta en este ejemplo.

- La calendarización de los eventos es asociada a cada persona que participa en el procedimiento.
TABLA III

Hipersensibilidad de las personas P1...P6

\begin{tabular}{|l|l|l|l|l|l|l|}
\hline Agente & P1 & P2 & P3 & P4 & P5 & P6 \\
\hline \hline Fenol & 3 & 3 & 3 & 3 & 3 & 3 \\
\hline Etanol & 1 & 1 & 1 & 1 & 1 & 2 \\
\hline Acetona & 2 & 1 & 1 & 1 & 2 & 1 \\
\hline Metanol & 1 & 1 & 1 & 1 & 1 & 1 \\
\hline Acetonitrilo & 1 & 1 & 1 & 1 & 1 & 1 \\
\hline Hexano & 1 & 2 & 1 & 1 & 1 & 1 \\
\hline $\begin{array}{l}\text { Ácido } \\
\text { tri cloro } \\
\text { acético }\end{array}$ & 1 & 1 & 1 & 1 & 1 & 1 \\
\hline Mercurio & 1 & 1 & 1 & 1 & 1 & 1 \\
\hline $\begin{array}{l}\text { Ácido per } \\
\text { clórico }\end{array}$ & 1 & 2 & 2 & 2 & 1 & 1 \\
\hline $\begin{array}{l}\text { Ácido } \\
\text { clor } \\
\text { hídrico }\end{array}$ & 1 & 2 & 3 & 2 & 1 & 1 \\
\hline \hline & & & & & \\
\hline
\end{tabular}

TABLA IV

Valores Umbral Límite de los agentes de interés (en PPM)

\begin{tabular}{|l|l|l|}
\hline Agente & $\begin{array}{l}\text { TLV- } \\
\text { TWA }\end{array}$ & $\begin{array}{l}\text { TLV- } \\
\text { STEL }\end{array}$ \\
\hline \hline Fenol & 5 & 5 \\
\hline Etanol & 500 & 1000 \\
\hline Acetona & 220 & 250 \\
\hline Metanol & 250 & 200 \\
\hline Acetonitrilo & 40 & 60 \\
\hline Hexano & 40 & 50 \\
\hline Ácido tricloroacético & 1 & 2 \\
\hline Mercurio & 0.25 & 0.25 \\
\hline Ácido perclórico & 0.227 & 0.454 \\
\hline Ácido clorhídrico & 1 & 2 \\
\hline \hline
\end{tabular}

2) Valores límite de los agentes:

Se detalla en la Tabla IV los valores límite (TLV) de los agentes químicos que se emanan en los eventos de la Tabla I.

\section{B. Modelo de Cálculo}

El modelo de cálculo propuesto está basado en la metodología utilizada en [13]. Se adaptó la metodología para usarla en el problema de evaluación de RQI. En la Figura 2 se muestran las etapas de cálculo de RQI individual.

En los términos referentes al cálculo debido a circunstancias estacionarias, primeramente se parte de una existencia dada en partes por millón (PPM) de cada uno de los agentes: $A_{1}, A_{2} \ldots A_{n}$. Posteriormente los modificadores se encargan de tomar en cuenta los factores de hipersensibilidad química individual, estado de salud, edad y años de servicio, al igual que la fracción de tiempo que la persona es expuesta. 


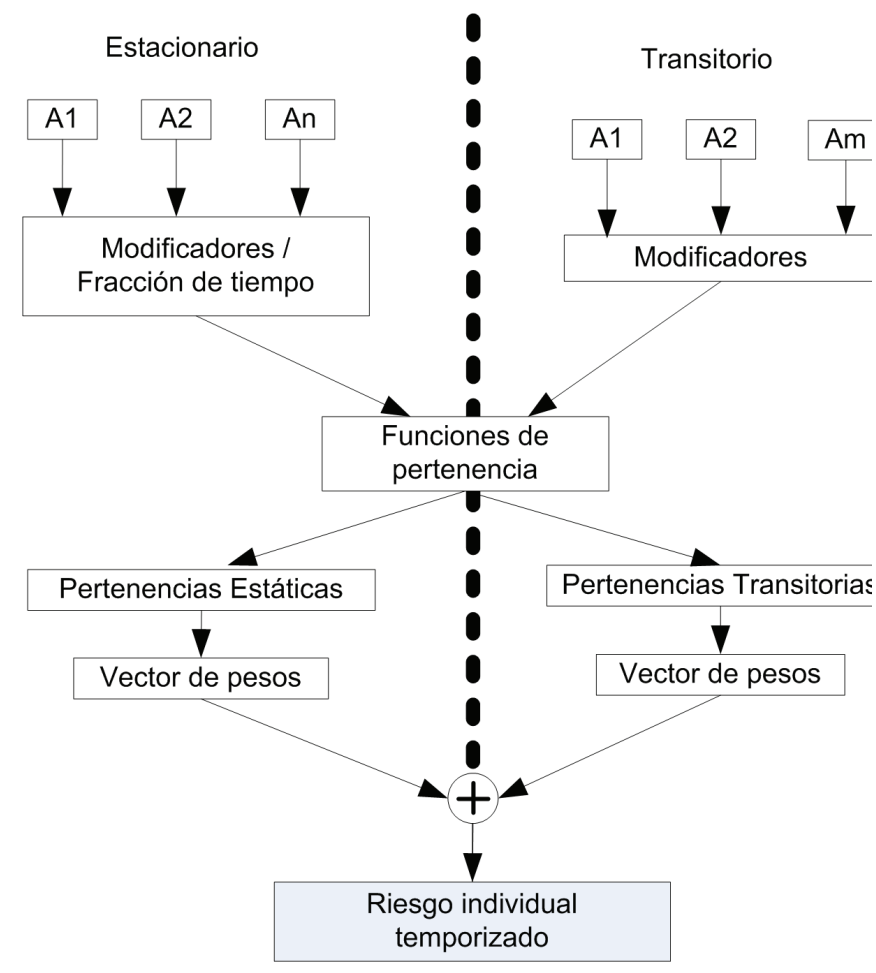

Fig. 2. Modelo de Cálculo Difuso.

Posteriormente, se procede a utilizar las funciones de pertenencia para determinar la ubicación difusa de cada uno de los agentes modificados. El resultado de esto corresponde a las pertenencias estáticas de clase, las cuales finalmente son sometidas a un cálculo de ponderación mediante un vector de pesos que permite obtener un número escalar que es indicador del RQI. Por otra parte, en los términos debido a circunstancias transitorias los agentes modificadores de las concentraciones PPM de cada agente no involucran una fracción de tiempo en el cálculo. El RQI individual temporizado corresponde a una superposición de ambos términos, el cual es mapeado en un conjunto de clases difusas que indican semánticamente el resultado final del cálculo.

A continuación se resume en seis pasos el procedimiento matemático que define el modelo propuesto.

\section{1) Definiciones básicas}

- $N$ la cantidad de agentes químicos tomados en cuenta en el modelo.

- $A_{E}[N]$ el vector cantidad en ppm de los agentes estacionarios, $A_{T}[N]$ el correspondiente para el caso transitorio. $A=A_{E} U A_{T}$.

- $M$ la cantidad (número impar) de clases difusas. Para este caso se define $M=7$.

2) Modificadores y fracción de tiempo

- La hipersensibilidad es una matriz $H_{2 N \times K}$ donde $K$ es la cantidad de personas expuestas y $H[n, k] \in$ $[0,5]$, según se expuso en la Tabla III.

- La salud es un vector $S_{K}$ donde $K$ es la cantidad de personas expuestas y $S[k] \in[0,10]$, según se expuso en la Tabla II.

- El vector $P_{K}$ es el que representa el modificador de la edad $e$ y los años de servicio $l$. Para la persona $k$ es obtenido mediante la ecuación:

$$
P(e, l)=0.01\left[e^{0.01\left(e_{k}-18\right)}-1\right]+0.05\left[e^{0.15\left(l_{k}\right)}+1\right]
$$

- La fracción de tiempo aplica para el caso de los agentes estacionarios, representada por un vector $F T_{K}$, donde cada $F T[k]$ contiene la fracción de una jornada de 8 horas en la que la persona está expuesta. $F T[k] \in[0,1]$.

3) Funciones de pertenencia.

La función de pertenencia del agente $i$ a la clase difusa $j$ está dada por $(0<j \leq 6)$ :

$$
u_{i, j}(x)=\left\{\begin{array}{cccc}
1-u_{i, j-1} & \text { si } & x \leq S_{i, j} \\
\frac{S_{i, j+1}-x}{S_{i, j+1}-S_{i, j}} & \text { si } & S_{i, j}<x \leq S_{i, j+1} \\
0 & \text { si } & x>S_{i, j+1}
\end{array}\right.
$$

Para $j=0, u_{i, 0}(x)=\frac{-1}{S_{i, 0} x+1} \cdot S_{i, 4}$ es el valor $T L V-T W A$

o TLV-STEL. Y $S_{i, j}=S_{i, 4}\left(1+\frac{j-4}{3}\right)$.

4) Entrada de la función de pertenencia.

Las funciones de pertenencia permiten calcular la disgregación de conjuntos para estimar cuál clasificación de riesgo previa debe darse a cada factor de entrada, tomando en cuenta los modificadores y la fracción de tiempo respectiva. De esta manera, las ecuaciones 3 y 4 muestran el cálculo para los agentes estacionarios y transitorios respectivamente. Esto para la persona $k$ y para el agente $i$.

$$
\begin{aligned}
& B_{E}[k][i]=A_{E}[i] H[k][i] S[k] P[k] \\
& B_{T}[k][i]=A_{T}[i] H[k][i] S[k] P[k] F T[k]
\end{aligned}
$$

La pertenencia de cada entrada se calcula con la ecuación 2 con las entradas dadas por las ecuaciones 3 y 4 . Así:

$$
\begin{aligned}
& u_{i, j}\left(B_{E}[k][i]\right) \\
& u_{i, j}\left(B_{T}[k][i]\right)
\end{aligned}
$$

Con $i: 1 . . N, k: 1 . . K, j: 0 . . M$.

5) Vector de pesos.

El factor de peso se debe calcular de manera dinámica, para cada instante en el que exista un evento transitorio, es decir 1 $\leq i \leq 2 N$ en todo $t$. Asimismo, el cálculo se debe realizar para la persona $k$. 


$$
\omega_{i, k}=\frac{\frac{B[k][i]}{m} \sum_{j=1}^{M} S_{i, j}}{\sum_{i=1}^{2 N} \frac{B[k][i]}{\sum_{j=1}^{M} S_{i, j}}}
$$

\section{6) Desfuzificación.}

La desfuzificación utiliza los pesos obtenidos en la ecuación 7 y los resultados de las pertenencias en las ecuaciones 5, 6 para obtener un número escalar para cada persona $k$. El resultado corresponde a $R[k] \in[0,7]$. Si, por ejemplo $R=3.47$, esto significa que el riesgo se encuentra en la categoría 3 .

\section{Resultados}

En esta sección se muestra los resultados de aplicar al modelo el conjunto de datos de prueba sometidos a una generación aleatoria de un calendario de realización de los eventos de la Tabla I de un total de 68 días hábiles. Asimismo, cada evento es asignado a una persona. La generación de reactivos químicos y la cantidad emanada se mantiene en concordancia con el evento respectivo. El objetivo de la simulación fue visualizar un número escalar dependiente del tiempo que muestre la evolución de la valoración de RQI tanto para cada persona como para la instalación en general.

\section{A. Resultados de la simulación para cada persona}

Los resultados para la persona 1 se muestran en la Fig. 3. Se puede destacar, por ejemplo, que esta persona presentó un riesgo superior al aceptable en 2 ocasiones en la simulación. Los resultados para las demás personas se omiten en este documento por razones de espacio.

\section{B. Resultados de la simulación para el laboratorio}

Los resultados globales se muestran en la Fig. 4. Éstos son resultado de una operación Global $[t]=\operatorname{Max}_{k}[t][k]$, de manera tal que cuando una única persona muestre un riesgo alto, esto bastará para clasificar de esta manera a la instalación. Los resultados globales contemplan la simulación realizada para las seis personas estudiadas en el ejemplo. Se puede observar que aquellos puntos que se acerquen al umbral de riesgo 5 o superiores serían catalogados como circunstancias en las que el RQI debe ser estudiado para encontrar los factores que ocasionaron su incremento.

\section{CONCLUSIONES}

La lógica difusa presenta un enfoque de modelado apropiado para el abordaje del problema de cálculo del RQI. Fue posible discernir en cada caso, las situaciones de exposición que provocan un riesgo significativo en las personas. Los modelos de valoración de riesgo estudiados hasta el momento, no toman en cuenta elementos como los que hemos denominado factores modificadores.

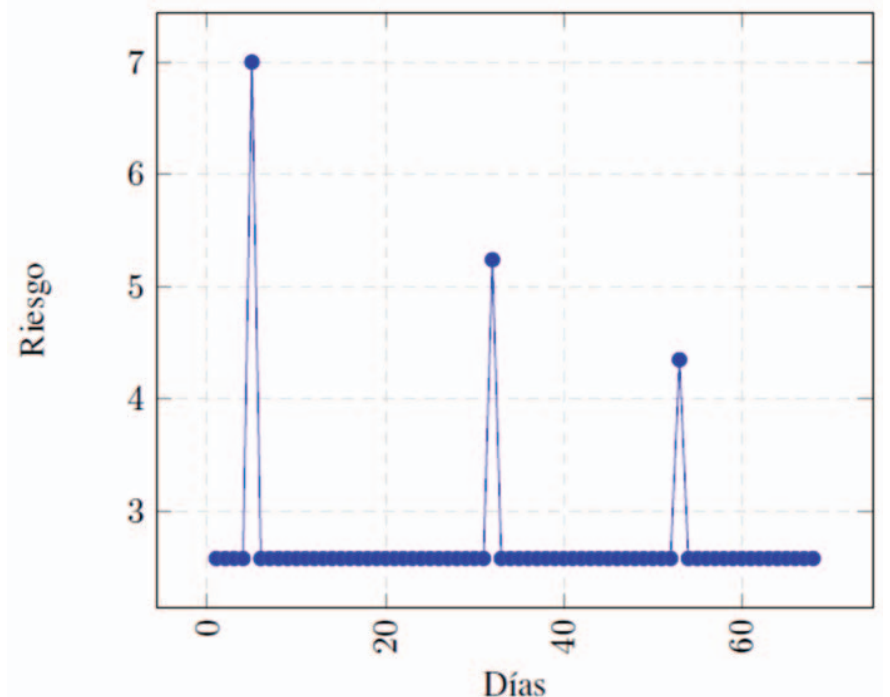

Fig. 3. Riesgo resultante: Persona 1.

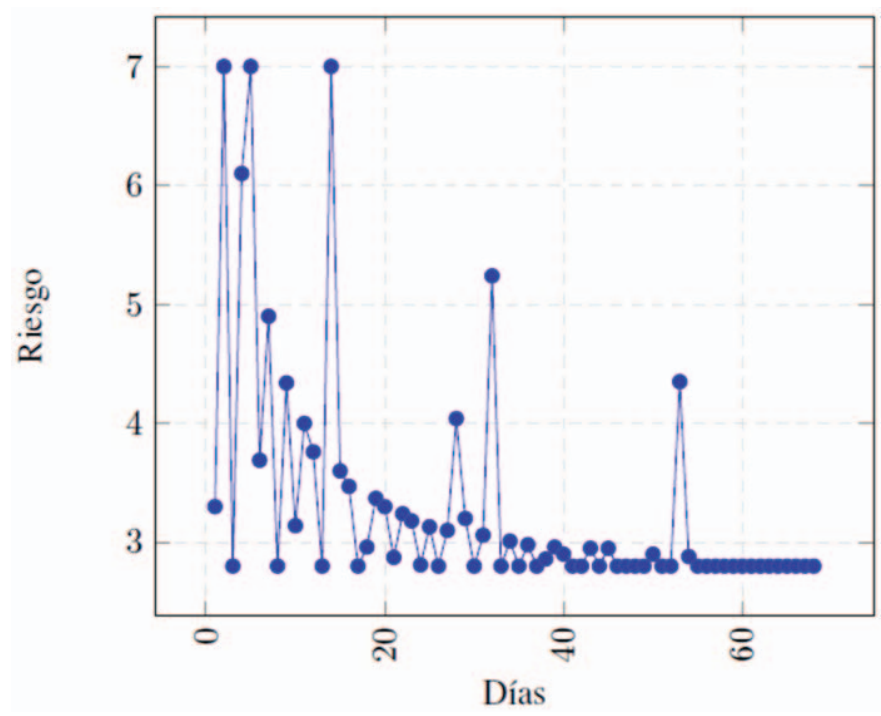

Fig. 4. Riesgo resultante: Caso global.

El modelo que se ha presentado, demuestra que es posible especificar con suficiente detalle estos elementos modificadores, para dirigir los esfuerzos de prevención, hacia cada persona temporal y espacialmente. Los resultados obtenidos, tanto en el RQI individual como en el RQI de la instalación evidencian la dependencia del tiempo y la plausibilidad del modelo planteado. Asimismo, la abstracción de la hipersensibilidad y demás modificadores individuales demostró su utilidad en los resultados respectivos.

\section{TRABAJO FUTURO}

En el presente artículo se han utilizado funciones de pertenencia simétricas y simples lineales. Debe profundizarse el estudio con datos suficientes para determinar cuál es el mejor abordaje en este aspecto.

La asignación de funciones de modificación para los factores de salud, edad y años laborados se definió de manera heurística, mediante una representación de peso exponencial. Debe ampliarse la visión de estos factores mediante la 
incorporación de estudios en toxicología, salud laboral e historiales médicos relacionados con sensibilidad química.

Los estimadores de pesos se encuentran basados en un criterio de mayor peso a los factores que presentan mayor riesgo. Debe determinarse si existe alguna manera más adecuada de obtener dichos factores.

El modelo propuesto en esta investigación será validado en al menos un laboratorio químico real, en una universidad costarricense. Otros tipos de modelado como el enfoque estocástico o de redes bayesianas para abordar el problema de cálculo de RQI pueden ser analizados.

\section{AgRADECIMIENTO}

Los autores desean manifestar su agradecimiento a la química Wendy M. Umaña Herrera, quien colaboró en la elaboración del conjunto de pruebas del modelo propuesto y en la documentación de los TVL utilizados.

\section{REFERENCIAS}

[1] R. Z. K. Karimpour, M.A. Moosavian and H. Bahmanyar, "New Fuzzy Model for Risk Assessment Based on Different Types of Consequences," Oil \& Gas Science and Technology - Rev. IFP Energies nouvelles, 2014.

[2] J. H. Duffus, Evaluación de riesgos químicos. Riesgos humanos, riesgos ambientales y riesgos ecológicos, 3rd ed. Montevideo, Uruguay: OMS, 1999.

[3] A. S. Markowski and M. S. Mannan, "Fuzzy risk matrix," Journal of hazardous materials, vol. 159, pp. 152-157, 2008.

[4] S.-M. Wang, T.-N. Wu, Y.-J. Juang, Y.-T. Dai, P.-J. Tsai, and C.-Y. Chen, "Developing a Semi-Quantitative Occupational Risk Prediction Model for Chemical Exposures and Its Application to a National Chemical Exposure Databank," International journal of environmental research and public health, vol. 10, pp. 3157-3171, 2013.

[5] M. E. Sousa, "Agentes químicos: evaluación cualitativa y simplificada del riesgo por inhalación," Instituto Nacional de Seguridad e Higiene en el Trabajo, España, Guía Técnica 937, 2012.

[6] X. G. Solá, "Regulación UE sobre productos químicos II. Reglamento CLP: Aspectos Básicos," Instituto Nacional de Seguridad e Higiene en el Trabajo, España, Guía Técnica 878, 2010.

[7] N. Caballé, "Agentes químicos: evaluación cualitativa y simplificada del riesgo por inhalación," Instituto Nacional de Seguridad e Higiene en el Trabajo, España, Guía Técnica 935, 2012.

[8] Cosanta. (2015). Stoffenmanager. Available: https://stoffenmanager.nl/default.aspx

[9] O. Y. Abul-Haggag and W. Barakat, "Application of Fuzzy Logic for Risk Assessment using Risk Matrix," International Journal of Emerging Technology and Advanced Engineering, vol. 3, 2013.

[10] W. Karwowski and A. Mital, "Potential applications of fuzzy sets in industrial safety engineering," Fuzzy sets and systems, vol. 19, pp. 105120, 1986.

[11] L. A. Zadeh, "Fuzzy sets," Information and control, vol. 8, pp. 338-353, 1965.

[12] D. A. Swayne, W. Yang, A. Rizzoli, and T. Filatova, "Modeling for Uncertainty Assessment in Human Health Risk Quantification: A Fuzzy Based approach," presented at the International Congress on Environmental Modelling and Software, Ottawa, Canada, 2010.

[13] W. Shi-Zhong, Z. Zhi-Hao, X. Bing, Q. Hao, J. Morel, and Q. RongLiang, "A fuzzy-based methodology for an aggregative environmental risk assessment of restored soil," Pedosphere, vol. 24, pp. 220-231, 2014.

[14] S. Bonvicini, P. Leonelli, and G. Spadoni, "Risk analysis of hazardous materials transportation: evaluating uncertainty by means of fuzzy logic," Journal of hazardous materials, vol. 62, pp. 59-74, 1998.

[15] ACGIH, TLVs and BEIs Book, 2015.

[16] F. M. McNeill and E. Thro, Fuzzy logic: a practical approach: Academic Press, 2014.

[17] M. I. Luster and G. J. Rosenthal, "Chemical agents and the immune response," Environmental health perspectives, vol. 100, p. 219, 1993.

\section{BIOGRAFÍAS}

Oscar M. Obando R. es Ingeniero en Electrónica, actual estudiante de la Maestría en Ciencias de Computación del Instituto Tecnológico de Costa Rica (ITCR). Actualmente es ingeniero de investigación y

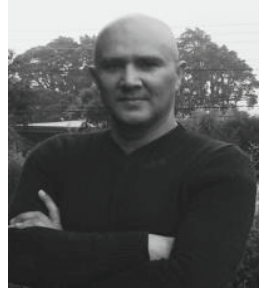
desarrollo en Hewllett Packcard y ha desarrollado en su vida profesional los temas de reconocimiento de patrones, inteligencia artificial y control automático. Ha sido profesor de ingeniería en diversas universidades en Costa Rica. Sus áreas de interés incluyen inteligencia artificial, sistemas predictivos, control automático y modelado matemático y estadístico.

César Garita es Ingeniero en Computación, Máster en Ciencias de la

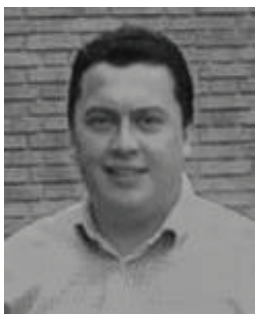
Computación del Instituto Tecnológico de Costa Rica (ITCR), y Doctor en Ciencias de la Computación de la Universidad de Ámsterdam (UvA), Países Bajos. Actualmente, es profesor catedrático del ITCR, cuenta con más de 70 publicaciones y es miembro de comités científicos de diferentes conferencias y revistas internacionales. Las áreas de interés del Dr. Garita incluyen integración de información distribuida, redes colaborativas, e-learning y arquitectura de 